trimester of pregnancy, and the most dangerous period is very shortly before or during labour, when transplacental haemorrhage is fairly common. ${ }^{3}$ It is possible to distinguish foetal cells amid a population of adult red cells by virtue of the ability of foetal haemoglobin to resist acid elution. If a fixed blood film is incubated for five minutes at $37^{\circ} \mathrm{C}$. in a

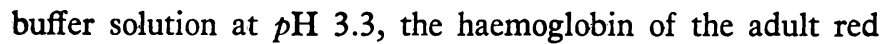
cells is lysed, whereas the foetal cells remain well coloured. ${ }^{4}$ The greater the number of foetal cells present in the mother's blood after delivery, the greater is the risk of subsequent immunization. ${ }^{3}$

In this week's B.M.f. a report from five centres describes the results of a trial designed to prevent $\mathrm{Rh}$-haemolytic disease. Earlier work by Professor C. A. Clarke and his colleagues had shown that $\mathrm{Rh}$-negative male volunteers could be protected from immunization by $\mathrm{Rh}$-positive cells if they were given a high-titre anti-D serum soon after transfusion. ${ }^{56}$ This work has now been extended to Rh-negative primiparae who had given birth to Rh-positive infants. Those with an appreciable number of foetal red cells in their circulation were given $5 \mathrm{ml}$. of gammaglobulin containing a very high titre of incomplete anti-D within 36 hours of delivery. The course of their immune response was then compared with that of an equal number of controls. Among 78 treated mothers there was no certain case of immunization, whereas among the 78 controls there were 19 cases of immunization. Furthermore, none of the treated women who have so far borne a subsequent $\mathrm{Rh}$-positive baby has developed antibodies. The mode of action of the gammaglobulin is not certain; it may lead to the foetal cells being rapidly destroyed, or else it may inhibit the immune response directly.

It seems as if this method will be effective in preventing maternal Rh-immunization and therefore Rh-haemolytic disease. When it is remembered that the disease affects about 1 in 200 of all babies born in Britain ${ }^{7}$ the importance of this work will be realized. A problem to be solved will be the manufacture of the gammaglobulin in sufficient quantities.

\footnotetext{
Landsteiner, K., and Wiener, A. S., Proc. Soc. exp. Biol. (N.Y.), 1940, 43, 223.

2 Coombs, R. R. A., Mourant, A. E., and Race, R. R., Brit. F. exp. Path., $1945,26,255$.

3 Woodrow, J. C., et al., Brit. med. F., 1965, 1, 279.

- - and Finn, R., Brit. F. Haematol., 1966, 12, 297.

${ }_{5}$ Finn, R., et al., Brit. med. F., 1961, 1, 1486.

6 Clarke, C. A., et al., ibid., 1963, 1, 979.

Walker, W., and Murray, S., ibid., 1954, 2, 126.
}

\section{Adrenal Cortex in Health and Disease}

Knowledge of the function of the adrenal cortex in health and disease has advanced more in the last decade than in the previous 100 years. In the Lumleian lecture published on page 847 last week and page 914 this week in the B.M.f. Dr. C. L. Cope has reviewed the present state of this knowledge. Its rapid growth has been due largely to the evolution and intelligent application of sensitive and precise physicochemical methods for measuring adrenal steroids and their metabolites. Recent advances include a technique for determining the rate of secretion of cortisol and aldosterone using radioisotopes and a simple fluorometric method for measuring plasma cortisol. ${ }^{1}$

The essential regulatory functions of the adrenal cortex stem from the actions of two hormones, cortisol and aldosterone, each under separate control. The first half of the Lumleian lecture is devoted to cortisol and its effects. The role of cortisol may be described as permissive, assisting rather than initiating the processes with which it is concerned. Though it has profound effects on most metabolic and physiological functions of the body and is essential for maintaining life during stress, the action of cortisol within cells is still unknown. The stimulus for cortisol production is pituitary corticotrophin, secretion of which is activated in two different ways. Basal secretion is regulated by a sensitive feed-back mechanism, which depends on the circulating level of cortisol, and which is suppressed by exogenous cortisol-like steroids and by the cortisol secreted by autonomous adrenal tumours. Conversely, secretion of corticotrophin increases in adrenal failure, whether this be primary and persistent, as in Addison's disease, or secondary and transient, as after prolonged corticosteroid therapy. Completely overriding the feed-back mechanism is the pituitary-adrenal response of the pituitary and adrenals to stress, which is mediated via the hypo- thalamus. The effects of long-term corticosteroid therapy can be understood only in relation to these two modes of control, for each may be suppressed differentially and recovery of one may precede that of the other.

The integrity of the feed-back mechanism can now be tested with metyrapone. ${ }^{2}$ Results have shown that after shortterm therapy with corticosteroids, even in massive doses, recovery is rapid and complete. Even after one or more years of therapy it is usual for adrenal activity to revive within 48 hours, though full recovery may take up to five months or rarely may never be complete. Unfortunately tests of pituitary-adrenal function using metyrapone give little or no indication of the patient's capacity to respond to severe stress, for this may demand a far greater release of corticotrophin than does the restoration or maintenance of normal adrenal activity via the feed-back mechanism. Cope discusses this problem in the light of recent knowledge, and concludes that as yet there is little factual evidence to support the common assumption that there is a significant risk of adrenal failure after corticosteroid therapy. Mere records of shock or collapse in vulnerable subjects are not enough to prove adrenal failure; nor is a favourable response to massive corticosteroid therapy in such emergencies. Data are required on the plasma corticosteroid level at the time of collapse. This should be easier to obtain when the new methods are more generally available. Furthermore, it should be noted that while corticotrophin injections given to cover the withdrawal of corticosteroid therapy may assist the recovery of adrenal function they are unlikely to enhance and may even suppress the capacity of the pituitary to secrete corticotrophin under stress.

New methods of testing have been of much value also in the field of diagnosis of primary disorders of the pituitaryadrenal axis. For example, the normal diurnal variation in

\footnotetext{
1 Mattingly, D., F. clin. Path., 1962, 15, 374.

2 Liddle, G. W., Estep, H. L., Kendall, J. W., Williams, W. C., and
} Townes, A."W., F. clin. Endocr., 1959, 19,.875. 
plasma cortisol is abolished in Cushing's syndrome. If in a suspected case the morning plasma cortisol is found to be within the upper range of normal the test has been of little value; but a finding of the same level around midnight strongly supports the diagnosis. It may be difficult to ascertain whether a given case of Cushing's syndrome is due to bilateral adrenal hyperplasia from oversecretion of corticotrophin or to an adrenal tumour, benign or malignant, in which secretory function is autonomous. Suppression tests with large enough doses of dexamethasone will reduce the level of cortisol secretion in the former but not in the latter group. Again, in mild Addison's disease cortisol secretion can be within the normal basal range because the surviving remnant of adrenal cortex is working under maximal stimulation. The diagnosis can be confirmed by showing no increase of secretion in response to injections of long-acting corticotrophin.

The second half of the Lumleian lecture deals with aldosterone, with particular reference to its possible role in the pathogenesis of hypertension and oedema. The principal action of this hormone is to restrict sodium and increase potassium excretion, effects which can be measured in urine, sweat, and saliva. Aldosterone is formed in the zona glomerulosa of the adrenal cortex, the normal rate of secretion being about one-hundredth that of cortisol. This last factor, together with the great difficulty of measuring aldosterone in body fluids, has restricted studies in this field to a few research centres, and there is no early prospect of routine laboratory methods being developed as with cortisol. The stimuli for aldosterone secretion are loss of sodium and water from the body, as after heavy diuresis or haemorrhage, and excessive loading with potassium. Conversely, secretion is suppressed by a high intake of sodium, excessive water retention, and potassium depletion. Control seems to be independent of the pituitary and hypothalamus, and the mechanism is still not clear. There is, however, increasing evidence that angiotensin II may be the principal stimulating factor. This substance is formed by interaction between the enzyme renin and an alpha-2-globulin in plasma. Renin is secreted by the juxtaglomerular apparatus of the kidneys and is easier to measure in body fluids than angiotensin II.

In the initial stages of oedema formation aldosterone probably plays a minor and secondary role and oedema cannot be induced in healthy subjects by giving large amounts of the hormone. Other factors such as raised venous pressure or lowered plasma-protein osmotic pressure are more important. The role of aldosterone in the pathogenesis of hypertension is complex, for oversecretion may be primary, or secondary to the vascular disorder. In most cases of essential hypertension secretion of the hormone is not increased, but in primary aldosteronism (Conn's syndrome) it seems to be the primary factor, causing hypertension through its effects on electrolyte balance. Oedema is notably absent in this condition and secretion of renin is suppressed. However, in malignant hypertension due to renal artery stenosis there is usually an increase in secretion both of aldosterone and of renin, though the excessive aldosterone production is probably secondary to the raised level of renin. Aldosterone may then potentiate this form of hypertension but does not initiate it. Cope discusses the difficulties that can arise in distinguishing between primary and secondary aldosteronism. The problem has become complicated even further by reports of adrenal adenomas occurring in a few cases of renal artery stenosis. These may arise in response to prolonged adrenal stimulation by angiotensin II, in turn secondary to renal ischaemia. Such adenomas may eventually become autonomous. It is therefore important that the adrenal glands should be inspected during operations for renal artery stenosis.

\section{Cough and Cancer}

The Committee on Smoking and Health of the Royal College of Physicians pointed out in its report $^{1}$ that universal abstinence from smoking should not be necessary to prevent lung cancer if only the minority of susceptible subjects could be detected and persuaded not to smoke. The committee cited preliminary evidence that a smoker's cough might be a danger signal.

Three subsequent studies have added some weight to this suggestion. E. C. Hammond ${ }^{2}$ in a postal survey of physical complaints among nearly half a million American men found that over the subsequent two years more of those with cough suffered from lung cancer than of those who did not cough, the excess increasing with severity of the cough. In 1951 K. R. Boucot and her colleagues started a periodic radiological and symptomatic survey of 6,137 men in Philadelphia. In their latest report ${ }^{3}$ they show that cigarette smokers who reported having a cough at the beginning of the study had an attack rate of lung cancer over the subsequent ten years that was twice that of non-coughing smokers. And the difference in the incidence of cancer between coughers and noncoughers was greater for moderate than for heavy smokers. In a retrospective study of men and women dying of lung cancer in Northern Ireland compared with a control group of men dying from non-respiratory causes G. Dean ${ }^{4}$ calculated that the lung cancer mortality of smokers who were reported to have a morning cough three years before death was between three and five times greater than that of non-coughers. $\mathrm{He}$ also found the difference was greater in lighter than in moderate or heavy smokers.

In all these reports it remains uncertain whether the increased mortality from lung cancer of the coughers was because some of them already had early cancer or because some had premalignant bronchial metaplasia, such as that described by $O$. Auerbach and his colleagues, ${ }^{5}$ or the mucoid secretion which R. D. Passey ${ }^{6}$ believes to be the condition responsible for many lung cancers. The remarkably low incidence of bronchial metaplasia in ex-smokers ${ }^{5}$ and the rapid decline of lung cancer mortality observed by Richard Doll and Sir Austin Bradford Hill ${ }^{7}$ among cigarette smokers who stop smoking shows that precancerous changes may be reversible or are at least not inevitably progressive. But once malignant changes have started it may be too late to benefit from stopping smoking. Nor can cough be regarded as helpful in obtaining early diagnosis, for this can seldom be firmly established, even if suspected, before fatal metastases have occurred. Before a smoker's cough could be a useful

\footnotetext{
'Royal College of Physicians, Smoking and Health, 1962. Pitman Medical, London.

2 Hammond, E. C., Amer. F. publ. Hlth, 1964, 54, 11. Boucot, K. R., Cooper, D. A., Weiss, W., and Carnahan, W. J., 7.

4 Dean, G., Brit. med. F., 1966, 1, 1506.

Auerbach, O., Stout, A. P., Hammond, E. C., and Garfinkel, L., New Engl. F. Med., 1961, 265, 253.

- Passey, R. D., Lancet, 1962, 2, 107.

7 Doll, R., and Hill, A. B., Brit. med. F., 1964, 1, 1460.

Spratt, J. S., Spiut, H. J., and Roper, C. L., Cancer (Philad.), 1963, 16,687

10 Garland, L. H., Coulson, W., and Wollin, E., ibid., 1963, 16, 694.

10 Fletcher, C. M., Proc. roy. Soc. Med., 1965, 58, 918.
} 